\title{
Dual renormalization group flows in 4D
}

\author{
Steven Abel, ${ }^{1, *}$ Borut Bajc, ${ }^{2, \dagger}$ and Francesco Sannino ${ }^{3, *}$ \\ ${ }^{1}$ IPPP, Durham University, South Road, Durham, DH1 3LE, United Kingdom \\ ${ }^{2}$ J. Stefan Institute, 1000 Ljubljana, Slovenia \\ ${ }^{3} \mathrm{CP}^{3}$-Origins \& the Danish IAS, University of Southern Denmark, \\ Campusvej 55, 5230 Odense M, Denmark
}

(Received 12 November 2018; published 6 March 2019)

\begin{abstract}
We present a prescription for using the $a$ central charge to determine the flow of a strongly coupled supersymmetric theory from its weakly coupled dual. The approach is based on the equivalence of the scale-dependent $a$ parameter derived from the four-dilaton amplitude with the $a$ parameter determined from the Lagrange multiplier method with scale-dependent $R$ charges. We explicitly demonstrate this equivalence for massive free $\mathcal{N}=1$ superfields and for weakly coupled SQCD.
\end{abstract}

DOI: 10.1103/PhysRevD.99.065001

\section{INTRODUCTION}

Renormalization group (RG) flow of quantum field theories (QFTs) is thought to be irreversible. In two dimensions, this irreversibility is encompassed by the Zamalodchikov $c$-theorem, which states that one can define a monotonically decreasing parameter that interpolates between the central charges $c$ [1] of two conformal theories related by an RG flow. An equivalent parameter in four dimensions is Cardy's proposal of the $a$ anomaly, the coefficient of the Euler density in the trace of the energy momentum tensor [2].

In a remarkable paper [3], Komargodski and Schwimmer (KS) produced a general form for this coefficient to show that its value will inevitably decrease if a system goes from a UV to an IR fixed point. The method that was used in [3] is a cousin of 't Hooft anomaly matching, in the sense that a spectator dilaton field is introduced that compensates the anomaly and restores exact Weyl symmetry at all scales, which is spontaneously broken by a dilaton VEV. Using such a setup, the $a$ parameter can be deduced from the four-dilaton amplitude. The change in the $a$ parameter between fixed points, $a_{I R}-a_{U V}$, is then found to be always negative by relating it via the optical theorem to the cross section. Thus, the weak form of the $a$-theorem, that its value will decrease if a system flows from a UV fixed point to an IR one, can be considered proven. However, the strong version, namely that

\footnotetext{
s.a.abel@durham.ac.uk

†borut.bajc@ijs.si

¥sannino@cp3.sdu.dk
}

Published by the American Physical Society under the terms of the Creative Commons Attribution 4.0 International license. Further distribution of this work must maintain attribution to the author(s) and the published article's title, journal citation, and DOI. Funded by SCOAP ${ }^{3}$. there exists a monotonically decreasing $a$ function with unambiguous physical meaning all along the flow, appears to be still open because of the presence of scheme-dependent $\beta^{2}$ terms in the four dilaton amplitude, as discussed in [4-6].

Indeed, Jack and Osborn [7-9] showed the existence of a function $\hat{a}$ related to $a$ through the beta functions, that coincides with it at fixed points and that flows with energy scale $\mu$ as

$$
\mu \frac{d \hat{a}}{d \mu}=\chi_{I J} \beta^{J} \beta^{I}
$$

where $\beta^{I}$ are the beta functions of couplings $\lambda_{I}$, and $\chi_{I J}$ is a metric on the space of couplings. The problem of proving the monotonicity of the function $\hat{a}$ (and, hence, the irreversibility of RG flow) is then reduced to one of proving the positive-definiteness of the metric $\chi$ on the space of functions. This problem remains to be solved (for a review see e.g., [10]).

Our purpose here is to point out that the parametric closeness of $a$ and $\hat{a}$ suggests a method of tracking the approximate flow of a strongly coupled theory. Indeed, generally, for flow between nearby fixed points, it seems natural to attempt a perturbative expansion in terms of the beta functions rather than in terms of any couplings [11]. In this article, we explore the $a$ parameter as the basis for such an approach, showing how one can use it to follow the flow of arbitrarily strongly coupled SQCD theories between fixed points.

Central to this approach is of course the fact that it is already known how to map the particle content of strongly coupled "electric" SQCD theories to weakly coupled "magnetic" ones via Seiberg duality $[12,13]$. Thus, one can already determine all the discrete parameters of strongly coupled theories, as well as much of their holomorphic data, even when they are away from fixed 
points. The question we will address here is how one can also determine the flow of the coupling in the strong theory, up to the aforementioned corrections of order $\beta^{2}$, by mapping from the weak theory.

The approach continues in the spirit of 't Hooft anomaly matching, by considering the flow of the $a$ parameter. In order to define such a flow, we will use the KS determination of $a$ which involves a certain integration of the fourdilaton amplitude over the Mandelstam variable [14]

$$
a_{U V}-a_{K S}(\mu)=\frac{f^{4}}{4 \pi} \int_{s>\mu^{2}} \frac{\operatorname{Im} \mathcal{A}(s)}{s^{3}} d s,
$$

where $f$ is the dilaton decay constant and $s$ is the Mandelstam variable, and where we impose an IR cutoff on the integral, $s>\mu^{2}$, in order to generate a running $a$ parameter, which we denote $a_{K S}(\mu)$. The cutoff induced scale dependence in the $a$ parameter interpolates its value smoothly and monotonically between its fixed point values. If one supposes that there exist dual descriptions of the entire flow between the UV and IR fixed points, then the flow induced in the $a$ parameter in the dual theories is identical by the above prescription.

The route from the $a$ parameters to the couplings is via $R$-charges and, hence, anomalous dimensions. Indeed, at the fixed points there already exist well known relations between the anomalous dimensions of fields, their $R$-charges (via the superconformal field theory), and the $a$ and $c$ parameters. The latter relations e.g., take the form ${ }^{1}$

$$
\tilde{a}=3 \operatorname{Tr} R^{3}-\operatorname{Tr} R ; \quad \tilde{c}=9 \operatorname{Tr} R^{3}-5 \operatorname{Tr} R,
$$

where here $R$ denotes the charges of states contributing to the 't Hooft anomalies (i.e., it would be $R-1$ if the superfield has charge $R$ ).

Thus, one prescription for defining a set of $R$-charges along the flow is to continue to solve (1.4) for $R(\mu)$ away from the fixed points, using $a_{K S}(\mu)$ as defined in (1.2). We should stress that such a prescription (and the anomalous dimensions it gives rise to) corresponds to a choice of renormalization scheme. However, as the right-hand side of (1.2) is the integral of a physical quantity (namely, by the optical theorem, the four-dilaton cross section) this particular choice has a physical meaning which is similar to that of the "sliding scale" scheme $[15,16]$. Moreover, it is independent of perturbation theory, so it has the same interpretation irrespective of whether one is using the electric or magnetic formulation.

\footnotetext{
${ }^{1}$ Here and in the following we will interchangeably use $a$ and $\tilde{a}$ related by
}

$$
a=\frac{3}{32(4 \pi)^{2}} \tilde{a}
$$

A second reason to favor "flowing" $R$-charges defined in such a way is that they appear to coincide with those of the Lagrange-multiplier method suggested by Kutasov $[17,18] .^{2}$ The starting point of our discussion will be to demonstrate this unexpected equivalence, for flows near fixed points in the Banks-Zaks limit. This gives some physical meaning to the Lagrange-multiplier method when the theory is strongly coupled. Remarkably the RG-scheme implicit in applying (1.4) to (1.2), appears to correspond to that implicit in the Lagrange-multiplier technique. ${ }^{3}$

Consequently one can determine the $R$-charges of the strongly coupled theory from those of the weakly coupled theory, by way of the matched $a$ parameters, which have a well-defined physical meaning in terms of the four-dilaton amplitude, independent of whether the description is strongly or weakly coupled. From there it is straightforward to determine the anomalous dimensions; hence, the NSVZ beta function, and ultimately the gauge coupling in the strongly coupled description.

\section{DILATON SCATTERING $a$ VERSUS LAGRANGE MULTIPLIER $a$}

Let us begin by showing (in the Banks-Zaks limit) that the $a(\mu)$ parameter one extracts for SQCD at scale $\mu$ along the flow between two fixed points using the KS definition [14] coincides with the Lagrange multiplier $a$ parameter of [17].

First consider $a_{K S}(\mu)$ in more detail. The prescription of (1.2) can be understood in terms of the contour integral of $\mathcal{A} / s^{3}$ around the loop shown in Fig. 1, where the radius of the inner contour is $\mu^{2}$. The amplitude in this integral is treated as holomorphic in the upper half-plane of complex $s$, with branch cuts arranged along the real axis. The integral in (1.2) corresponds to going along the $I_{2}$ portion of the contour above the branch cuts of the amplitude which run along the real axis to plus infinity in the $s$ channel (and minus infinity in the $u$-channel). In the IR, the amplitude behaves as

$\mathcal{A}(s)=8\left(a_{U V}-a_{I R}\right) \frac{s^{2}}{f^{4}}+\mathcal{O}\left(\frac{m^{2\left(4-\Delta_{I R}\right)} s^{\Delta_{I R}-2}}{f^{4}}\right)$,

where $\Delta_{I R}>4$ is the lowest dimension of the irrelevant operators (of the dilaton) in the IR theory, and hence $m$ is the scale of the relevant operators that we added into the UV theory that generated them upon integrating out degrees of freedom. In the limit that $\mu \rightarrow 0$, we may simply neglect the terms with inverse powers of $m$ (along with $I_{3}$ which tends to zero) and performing the integral find by Cauchy's theorem [4],

\footnotetext{
${ }^{2}$ This method relies on there being a Lagrangian description of the theory, which will be assumed in the following.

${ }^{3}$ If along the flow a gauge invariant operator becomes free, a new accidental symmetry arises and one should properly define $a_{K}(\mu)$ along the lines of [19].
} 


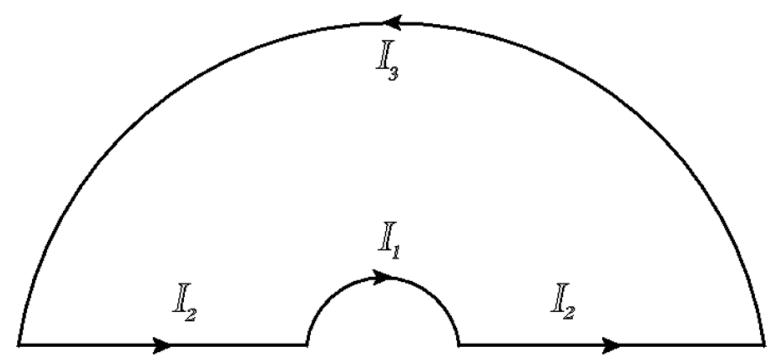

FIG. 1. Contour for $a_{K S}(\mu)$.

$-I_{1}=\frac{8 \pi\left(a_{U V}-a_{I R}\right)}{f^{4}}=I_{2}=2 \int_{0}^{\infty} d s \frac{\operatorname{Im} \mathcal{A}(s)}{s^{3}}$,

where we also require Schwartz reflectivity of the amplitude (namely $\overline{\mathcal{A}(s)}=\mathcal{A}(\bar{s})$ ). This (by way of the optical theorem) is enough to establish the weak $a$-theorem.

By contrast at finite $\mu$ the answer for $I_{1}$ is, of course, $\mu$ dependent. To demonstrate what happens let us first revisit the simple example of free scalar fields of mass $m$ discussed in [3]. Using standard perturbation theory (with the conventions of [3]) their contribution to the four-dilaton amplitude is found to be

$$
\begin{aligned}
\mathcal{A}= & -240\left(a_{U V}-a_{I R}\right) \\
& \times \frac{m^{4}}{f^{4}} \int_{0}^{1} d x\left(\log \left(m^{2}-s x(1-x)\right)\right. \\
& \left.+\log \left(m^{2}+s x(1-x)\right)\right)+ \text { const },
\end{aligned}
$$

where we assume only these fields contribute to $a_{U V}-a_{I R}$. The constant term is independent of $s$ and contains counter-terms to remove infinities, but it is not important for the discussion. Expanding the logarithms in $\mathrm{s} / \mathrm{m}^{2}$ and performing the $x$ integral gives the leading contribution in (2.1) (which can be used to check the prefactor). Alternatively, we note that the new absorptive contribution to $\mathcal{A}$ comes from the region of the integral where the argument of the first logarithm is negative, $s x(1-x)>m^{2}$. Taking $s \rightarrow s+i \epsilon$ in order to be above the branch cuts, we find

$$
\operatorname{Im} \mathcal{A}=240 \pi\left(a_{U V}-a_{I R}\right) \frac{m^{4}}{f^{4}} \sqrt{1-4 m^{2} / s} .
$$

Inserting this into the integral $I_{2}$ with a cutoff then gives a running $a$ parameter

$$
\begin{aligned}
a_{U V}-a_{K S}(\mu) & =\frac{f^{4}}{4 \pi} \int_{s>\mu^{2}} \frac{\operatorname{Im} \mathcal{A}(s)}{s^{3}} d s, \\
& =\left(a_{U V}-a_{I R}\right)(1-\rho(\mu / 2 m)),
\end{aligned}
$$

where

$$
\rho(x)= \begin{cases}\left(1-x^{-2}\right)^{3 / 2}\left(1+\frac{3}{2} x^{-2}\right) & ; \quad x \geq 1 \\ 0 & ; \quad x \leq 1 .\end{cases}
$$

For later comparison, it is useful to rearrange the expression as

$$
a_{K S}=a_{I R}+\left(a_{U V}-a_{I R}\right) \rho(\mu / 2 m),
$$

making it clear that $\rho(\mu / 2 m)$ correctly scales the contributions of the scalars to the $a_{K S}$ parameter continuously and monotonically, with $\rho=0$ at $\mu=2 m$ to $\rho=1$ at $\mu \rightarrow \infty$. Thus, we may interpret the KS integral of (1.2) as simply counting the imaginary (absorptive) contributions to the amplitude from states that are able to go on shell when $s>\mu^{2}$ (a useful reference in this context is [20]).

In order to compare the running $a_{K S}$ derived above with the continuously varying $a_{K}$ function devised for SUSY theories in [17], we need to extend the simple case above to $\mathcal{N}=1$ SUSY. Consider the free field theory, consisting of $N_{f}$ pairs of superfields $\Phi^{a}$ and $\tilde{\Phi}^{a}, a=1 \ldots N_{f}$. The Lagrangian of [3] can be made supersymmetric in the obvious way, by coupling the fields in a superpotential mass-term $W \supset m \Omega \tilde{\Phi} \mathbb{I}_{\Delta_{f} \times \Delta_{f}} \Phi$, where $\Omega f$ is the canonically normalized dilaton superfield with $\langle\Omega\rangle=1$. This gives a supersymmetry preserving mass $m$ to $\Delta_{f}$ pairs of superfields. (As the superpartner of the dilaton does not appear in any loops of interest, we can ignore it.)

The amplitude is, of course, augmented by superpartner diagrams, but now supersymmetry guarantees that the coefficient of terms such as (2.3) vanish because, otherwise (as these terms are not zero in the limit of vanishing external momenta and finite $f$ ), they would signal a renormalization of the superpotential. The nonvanishing terms of interest are, in the standard Passarino-Veltman notation, of the form $s \mathcal{B}_{0}\left(s, m^{2}, m^{2}\right)$ and friends. Thus, the contributions of interest are of the form

$$
\begin{aligned}
\mathcal{A}= & -24\left(a_{U V}-a_{I R}\right) \frac{m^{2} s}{f^{4}} \int_{0}^{1} d x\left(\log \left(m^{2}-s x(1-x)\right)\right. \\
& \left.-\log \left(m^{2}+s x(1-x)\right)\right)+\mathrm{const}
\end{aligned}
$$

where as before the second term is really the $u$ Mandelstam variable with $t \rightarrow 0$.

Following the above treatment of the massive scalar, we deduce a running $a_{K S}$ from the absorptive part which is

$$
\operatorname{Im} \mathcal{A}=24 \pi\left(a_{U V}-a_{I R}\right) \frac{m^{2}}{f^{4}} s \sqrt{1-4 m^{2} / s}
$$

Inserting this into (1.2) then gives (2.7), but with a modified scaling function, 


$$
\rho(x)= \begin{cases}\left(1-x^{-2}\right)^{3 / 2} & ; \quad x \geq 1 \\ 0 & ; \quad x \leq 1\end{cases}
$$

Let us now compare this expression with the $a_{K}$ function of [17]. The Lagrange multiplier method for this simple case goes as follows. In general, the running $a$ parameter is defined by adding a Lagrange multiplier for each relevant operator. In this case, there is only one of them, which imposes the constraint from the mass term. The $a$-function is therefore given simply by

$$
\begin{aligned}
\tilde{a}_{K}= & \left(N_{f}-\Delta_{f}\right)\left[3(R-1)^{3}-(R-1)\right] \\
& +\Delta_{f}\left[3(r-1)^{3}-(r-1)\right]+\lambda \Delta_{f}[r-1],
\end{aligned}
$$

where $R$ is the $R$-charge of the $N_{f}-\Delta_{f}$ chiral superfields that remain massless and $r$ is the $R$-charge of the last $\Delta_{f}$ flavors, which is considered to be a function of the energy scale. Thus, the $R$-symmetry we are following along the flow is a linear combination of the superconformal $R$-symmetry of the deep $U V$ and the $\mathrm{SU}\left(N_{f}\right) \times \mathrm{SU}\left(N_{f}\right)$ flavor symmetry with which it mixes because of the mass-term (specifically the $\operatorname{diag}\left(\Delta_{f} \mathbb{I}_{N_{f}-\Delta_{f}},\left(\Delta_{f}-N_{f}\right) \mathbb{I}_{\Delta_{f}}\right)$ component). ${ }^{4}$

One first solves to maximize the $a$ function with respect to unfixed $R$-charges, $\frac{\partial a}{\partial r}=\frac{\partial a}{\partial R}=0$. In the absence of the mass-term constraint, this simply chooses the free-field value of $2 / 3$ for both $R$ and $r$. However, at arbitrary Lagrange multiplier values one finds

$$
\begin{aligned}
& R=2 / 3, \\
& r=1-\frac{\sqrt{1-\lambda}}{3} .
\end{aligned}
$$

The case where $\lambda=0$ corresponds to $R=r=2 / 3$ in the deep UV, while $\lambda=1$ corresponds to $r=1$, which is the value forced upon it by the mass-term in the deep IR. Substituting these values into $\tilde{a}_{K}$ we have $\tilde{a}_{\mathrm{UV}}=\frac{2}{9} N_{f}$ and $\tilde{a}_{\mathrm{IR}}=\frac{2}{9}\left(N_{f}-\Delta_{f}\right)$, and a running $a$ parameter given by

$$
\tilde{a}_{K}=\tilde{a}_{\mathrm{IR}}+\left(\tilde{a}_{\mathrm{UV}}-\tilde{a}_{\mathrm{IR}}\right)(1-\lambda)^{\frac{3}{2}} .
$$

Comparison with (2.10) shows that the two $a$ functions precisely coincide if one makes the identification $\lambda \equiv \frac{4 m^{2}}{\mu^{2}}$. Note that the $a$ functions in the supersymmetric case match essentially because of the nonrenormalization theorem, and that as usual the Lagrange multiplier is essentially the "coupling" that induces the flow.

For the SUSY gauge theories of interest the situation is more complicated but the interpretation is always the same; namely $a_{K S}$ counts the physical states that are able to

\footnotetext{
${ }^{4}$ This is true for $\Delta_{f}<N_{f}$ : when $\Delta_{f}=N_{f}$ there is, of course, no relevant $R$-symmetry left.
}

contribute to the absorptive part of the four-dilaton amplitude. Meanwhile $a_{K}$ tracks the mixing of the UV $R$-symmetry with flavor symmetry along the flow [18]. We will now show that at weak coupling, close to the Caswell-Bank-Zaks fixed point, they are equivalent in this case as well. ${ }^{5}$

Consider SQCD with $N_{f}$ flavors of quarks $Q$ and $\tilde{Q}$ flowing from the asymptotically free theory to the fixed point. The $a_{K S}$ parameter was derived in terms of the gauge coupling in [14];

$$
a_{K S}(\mu)=a_{U V}-\frac{N_{c}^{2}}{128 \pi^{2}} \int_{g^{-2}(\mu)}^{\infty} \frac{d \lambda}{\lambda^{2}} \beta_{\lambda},
$$

where $\lambda=1 / g^{2}$. In the limit $\mu \rightarrow 0$, this expression reduces to Eq. (3.12) of [14]. Using

$$
b_{2}=\frac{N_{c} N_{f}}{\left(8 \pi^{2}\right)^{2}}, \quad g_{*}^{2}=\frac{8 \pi^{2}}{N_{f}} \epsilon,
$$

where

$$
\epsilon=\frac{3 N_{c}-N_{f}}{N_{c}} \ll 1
$$

the integral gives

$$
a_{K S}(\mu)=a_{U V}-\frac{N_{c}^{3} N_{f}}{32\left(8 \pi^{2}\right)^{3}} g^{2}(\mu)\left(2 g_{*}^{2}-g^{2}(\mu)\right),
$$

with $g^{2}(\mu)$ being a solution of the two-loop RGE,

$$
\frac{d g^{2}}{d \log \mu}=b_{2} g^{4}\left(g^{2}-g_{*}^{2}\right)
$$

Again, we can compare this parameter to the continuously varying $a_{K}$-function of [17]. In an $S U(N)$ gauge theory, it can be written in generality as

$\tilde{a}_{K}=2\left(N_{c}^{2}-1\right)+\sum_{i}\left|r_{i}\right|\left(a_{1}\left(R_{i}\right)-\left(R_{i}-R_{i}^{I R}\right) a_{1}^{\prime}\left(R_{i}\right)\right)$,

where $\left|r_{i}\right|$ is the dimension of the representation $r_{i}$, the prime means derivative with respect to $R$ and where

$$
a_{1}(r) \equiv 3(r-1)^{3}-(r-1)
$$

In the case of electric SQCD, this gives

\footnotetext{
${ }^{5}$ It would be interesting to look for reasons behind this equivalence that are valid beyond weak coupling, along the lines of $[21,22]$. For the present work the equivalence in the weakly coupled theories is sufficient.
} 
$\tilde{a}_{K}(\mu)=2\left(N_{c}^{2}-1\right)+2 N_{c} N_{f}\left(a_{1}\left(R_{Q}\right)-\left(R_{Q}-R_{Q}^{*}\right) a_{1}^{\prime}\left(R_{Q}\right)\right)$.

In order to compare with $a_{K S}$, we relate the $R$-charges to the anomalous dimensions through

$$
R_{Q}=\frac{2}{3}\left(1+\frac{\gamma_{Q}}{2}\right)
$$

This equation holds along the flow, but only at the endpoints of the flow does $R_{Q}$ coincide with the respective superconformal $R$-charges of the fixed points. The anomalous dimension can be perturbatively calculated at one loop as

$$
\gamma_{Q}=-\frac{N_{c}}{8 \pi^{2}} g^{2}
$$

and then using

$$
R_{Q}^{U V}=\frac{2}{3}, \quad R_{Q}^{*}=1-\frac{N_{c}}{N_{f}},
$$

we easily find the same leading contribution as that in (2.17), and hence $a_{K} \equiv a_{K S}$ as we wished to prove.

At this point one could ask, what is the meaning of equating a scheme-independent quantity such as $a_{K S}(\mu)$ with a scheme-dependent one such as $a_{K}(\mu)$. This is, of course, what we always do when we calculate a cross section (in which we are bound to choose a scheme), and compare it to its (scheme-independent) measured value. The theoretical result becomes scheme independent only when all terms in perturbation theory are taken into account, but never at finite order. Therefore the equivalence is only a perturbative one. Nevertheless as we shall now show, what it does do is allow us to develop a perturbative description of the flow in a strongly coupled theory.

\section{A PERTURBATIVE CALCULATION OF A NONPERTURBATIVE FLOW}

We now wish to explore how this equivalence can be used to determine the gauge coupling flow in a strongly coupled description. To do so we will consider a strongly coupled SQCD (in the conformal window) when one invokes a flow by adding a mass term for one flavor, and will make use of the well-known duality between this theory and Higgsing in a weakly coupled magnetic description, described in [12]. ${ }^{6}$

The original electric SQCD theory is an $\mathcal{N}=1 \mathrm{SU}\left(N_{c}\right)$ theory with $N_{f}+1$ flavors of $Q$ and $\tilde{Q}$ quarks and antiquarks. We add a mass-term of the form

$$
W_{e}=m Q_{N_{f}+1} \tilde{Q}^{N_{f}+1}
$$

\footnotetext{
${ }^{6}$ For a pedagogical description of such a setup, see e.g., [23].
}

TABLE I. The dual theories considered in the text with $N_{c}=N_{f}-\tilde{N}_{c}$. We consider throughout the case of $N_{f}=\frac{3}{2} N_{c}+\frac{1}{2}=3 \tilde{N}_{c}-1$.

\begin{tabular}{lll}
\hline \hline & $\operatorname{IR}(t<0)$ & $\mathrm{UV}(t>0)$ \\
\hline Magnetic Theory & $N_{f}$ flavors & $N_{f}+1$ flavors \\
& $\tilde{N}_{c}$ colors & $\tilde{N}_{c}+1$ colors \\
Electric Theory & $N_{f}$ flavors & $N_{f}+1$ flavors \\
& $N_{c}$ colors & $N_{c}$ colors \\
\hline \hline
\end{tabular}

in its superpotential. In the IR, i.e., at energy below $m$, it flows to a new theory with $N_{f}$ flavors; hence, effectively, there is a UV fixed point with $N_{f}+1$ flavors at energy above $m$, and an IR fixed point with $N_{f}$ flavors. If we take $2 N_{f}=3 N_{c}+1$ then the theory is expected to be strongly coupled for large $N_{c}$ all along the flow.

Meanwhile the magnetic description is an $\mathrm{SU}\left(\tilde{N}_{c}+1\right)$ theory with $\tilde{N}_{c}=N_{f}-N_{c}$ and, as well as $N_{f}+1$ flavors of quarks $q$ and $\tilde{q}$, it contains an elementary $\left(N_{f}+1\right) \times$ $\left(N_{f}+1\right)$ meson $\Phi$ formed from a composite of the electric quarks, which we will take to be $\Phi \equiv \frac{1}{\Lambda} Q \cdot \tilde{Q}$ where $\Lambda$ is the dynamical scale of the theory, and a superpotential

$$
W_{m}=m \Lambda \Phi_{N_{f}+1}^{N_{f}+1}+\tilde{y} \Phi \tilde{q} \cdot q,
$$

whose first term derives from the mass-term, and where the Yukawa coupling is $\tilde{y}=\Lambda / \hat{\Lambda}$ with $\hat{\Lambda} \sim \Lambda$. The magnetic theory which has $N_{f}=3 \tilde{N}_{c}-1$ is arbitrarily weakly coupled, so its flow can be followed perturbatively. In particular the linear meson term in the superpotential causes a Higgsing down to $\mathrm{SU}\left(\tilde{N}_{c}\right)$. For completeness we summarize the flows as seen in the two dual theories in Table I, where the RG scale is defined with respect to $m$, that is

$$
t \equiv \log (\mu / m)
$$

We can easily determine the difference between the UV and IR $a$-central charges

$$
\begin{aligned}
\tilde{a}_{\mathrm{UV}}-\tilde{a}_{\mathrm{IR}}= & 2 N_{c}\left(N_{f}+1\right) a_{1}\left(1-N_{c} /\left(N_{f}+1\right)\right) \\
& -2 N_{c} N_{f} a_{1}\left(1-N_{c} / N_{f}\right) \\
= & \frac{6 N_{c}^{2}\left(2 N_{f}+1\right)}{N_{f}^{2}\left(N_{f}+1\right)^{2}}
\end{aligned}
$$

which is positive for all $N_{f}>0$, and thus the weak $a$-theorem is satisfied.

As discussed, our aim is to determine the gauge coupling for the original strongly coupled electric theory. In order to do this, we first consider in detail the dual of the UV theory, and the dual of the IR theory, both of which are known. By choosing $N_{f} \approx 3 \tilde{N}_{c}$ and large $\tilde{N}_{c} \equiv N_{f}-N_{c}$, the magnetic theory ${ }^{7}$ is made perturbative both in the UV and the IR so 
we can calculate its flow with good accuracy along the whole RG trajectory. As we also know the (in principle nonperturbative) interacting electric theories in both the UV and the IR, we assume that the flow of the magnetic theory is dual to that of the strongly coupled electric theory along the whole trajectory.

Before entering into the explicit computation, let us clarify the idea and the procedure we will follow. The magnetic theory is perturbative and is, thus, under control in the whole region between the deep UV $(\mu=+\infty$, $t=+\infty)$ and the deep IR $(\mu=0, t=-\infty)$. The gauge and Yukawa couplings are continuous along the whole flow, while the two beta functions and the two anomalous dimensions are, due to the mass-independent character of the NSVZ scheme, discontinuous at the explicit quark mass scale $(\mu=m, t=0)$.

However, in one half of the flow $(\mu<m)$ we are able to use the $a$ parameters to track (perturbatively and in the NSVZ scheme) the evolution of the strongly coupled theory from that in the weakly coupled one. In particular, the electric theory is nonperturbative and what we know from Seiberg duality is that it is equivalent to the magnetic theory at $\mu=m(t=0)$, which becomes the "new" $\mathrm{UV}$, and in the deep IR at $\mu=0(t=-\infty)$. By continuity ${ }^{8}$ we assume that the perturbative magnetic and nonperturbative electric theories are dual between these two endpoints. In this region, the physical quantity $a_{K S}$ is by definition the same in the magnetic and electric theory, and as motivated in the previous section, we equate $a_{K}$ and $a_{K S}$, allowing us to explicitly calculate the central charge in the whole energy range $0<\mu<m$ in the magnetic theory, as a perturbative function of the gauge and Yukawa coupling constants. This (via the equivalence of the $a$ parameters) yields the nonperturbative $R$-charges and, hence, anomalous dimensions of the strongly coupled electric theory, which in turn yields the explicit numerical solution of the RGE for the electric gauge coupling constant.

\section{A. UV $(0<t<\infty)$ : Magnetic theory}

We start in the UV with the magnetic theory, which is an $\mathrm{SU}\left(\tilde{N}_{c}+1\right)$ gauge theory with $N_{f}+1$ quarks $q+\tilde{q}$ and $\left(N_{f}+1\right)^{2}$ singlet meson fields with the superpotential of (3.2). We will work in terms of

$$
\tilde{\alpha}_{g} \equiv \frac{\left(\tilde{N}_{c}+1\right) \tilde{g}^{2}}{(4 \pi)^{2}}, \quad \tilde{\alpha}_{y} \equiv \frac{\left(\tilde{N}_{c}+1\right) \tilde{y}^{2}}{(4 \pi)^{2}}
$$

The theory is asymptotically free when $N_{f}+1=3 \tilde{N}_{c}$ since

$$
b_{1}=3\left(\tilde{N}_{c}+1\right)-\left(N_{f}+1\right)=3 \text {, }
$$

and at $t \rightarrow \infty$ all couplings go to zero. For $\mu \gg \Lambda$, the one-loop approximation is sufficient, and one has the usual evolution with dynamical scale given by $\Lambda \equiv \mu \exp \left(-\frac{1}{2 b_{1} \tilde{\alpha}_{g}(\mu)}\right)$. Towards the IR the flow approaches a Banks-Zaks fixed point that for larger $\tilde{N}_{c}$ becomes increasingly perturbative. Indeed, the two-loop RGEs (see e.g., $[11,24])$ give the fixed points to be at

$$
\begin{aligned}
& \tilde{\alpha}_{g}\left(0^{+}\right)=\frac{\left(2 \frac{N_{f}+1}{\tilde{N}_{c}+1}+1\right)\left(\frac{N_{f}+1}{\tilde{N}_{c}+1}-3\right)}{6+8 \frac{N_{f}+1}{\tilde{N}_{c}+1}-4\left(\frac{N_{f}+1}{\tilde{N}_{c}+1}\right)^{2}+2 \frac{N_{f}+1}{\left(\tilde{N}_{c}+1\right)^{3}}} \stackrel{N_{f} \rightarrow 3 \tilde{N}_{c}-1}{\longrightarrow} \frac{7}{2 \tilde{N}_{c}}+\frac{43}{2 \tilde{N}_{c}^{2}}+\mathcal{O}\left(1 / \tilde{N}_{c}^{3}\right), \\
& \tilde{\alpha}_{y}\left(0^{+}\right)=\frac{2\left(1-\frac{1}{\left(\tilde{N}_{c}+1\right)^{2}}\right)\left(\frac{N_{f}+1}{\tilde{N}_{c}+1}-3\right)}{6+8 \frac{N_{f}+1}{\tilde{N}_{c}+1}-4\left(\frac{N_{f}+1}{\tilde{N}_{c}+1}\right)^{2}+2 \frac{N_{f}+1}{\left(\tilde{N}_{c}+1\right)^{3}}} \stackrel{N_{f} \rightarrow 3 \tilde{N}_{c}-1}{\longrightarrow} \frac{1}{\tilde{N}_{c}}+\frac{7}{\tilde{N}_{c}^{2}}+\mathcal{O}\left(1 / \tilde{N}_{c}^{3}\right) .
\end{aligned}
$$

Defining

$$
\Delta_{\tilde{g}}^{(+)}(t)=\tilde{\alpha}_{g}(t)-\tilde{\alpha}_{g}\left(0^{+}\right), \quad \Delta_{\tilde{y}}^{(+)}(t)=\tilde{\alpha}_{y}(t)-\tilde{\alpha}_{y}\left(0^{+}\right),
$$

the two-loop RGEs can be rephrased as

$$
\begin{aligned}
& \frac{d \tilde{\alpha}_{g}(t)}{d t}=-2 \tilde{\alpha}_{g}^{2}(t) \times\left[\left(6-4 \frac{N_{f}+1}{\tilde{N}_{c}+1}+2 \frac{N_{f}+1}{\left(\tilde{N}_{c}+1\right)^{3}}\right) \Delta_{\tilde{g}}^{(+)}(t)+2\left(\frac{N_{f}+1}{\tilde{N}_{c}+1}\right)^{2} \Delta_{\tilde{y}}^{(+)}(t)\right], \\
& \frac{d \tilde{\alpha}_{y}(t)}{d t}=2 \tilde{\alpha}_{y}(t) \times\left[-2\left(1-\frac{1}{\left(\tilde{N}_{c}+1\right)^{2}}\right) \Delta_{\tilde{g}}^{(+)}(t)+\left(2 \frac{N_{f}+1}{\tilde{N}_{c}+1}+1\right) \Delta_{\tilde{y}}^{(+)}(t)\right] .
\end{aligned}
$$

The terms proportional to $\tilde{\alpha}_{g}\left(0^{+}\right)$and $\tilde{\alpha}_{y}\left(0^{+}\right)$in these expressions are the one-loop terms, while the remaining terms are two-loop.

\footnotetext{
${ }^{7}$ It is convenient to take the magnetic theory to be perturbative as then only one coupling-the electric gauge coupling-is nonperturbative and, thus, the matching of $a$ parameters determines it uniquely.

${ }^{8}$ For a discussion on this point see e.g., [23].
} 
Finally, we can calculate the R-charges at the $t=0^{+}$ fixed point,

$R_{q}\left(0^{+}\right)=1-\frac{\tilde{N}_{c}+1}{N_{f}+1}, \quad R_{\Phi}\left(0^{+}\right)=2-2 R_{q}\left(0^{+}\right)$,

which are perturbative (free) $R_{q}=R_{\Phi}=2 / 3$ in the large $\tilde{N}_{c}$ limit with $N_{f} \rightarrow 3 \tilde{N}_{c}-1$, in accord with the magnetic theory being parametrically perturbative for all positive $t$.

\section{B. UV $(0<t<\infty)$ : Electric theory}

Apart from the far UV (as it is also asymptotically free), the form of the electric dual theory is known only in the $t \rightarrow 0^{+}$limit, where it is an $\mathrm{SU}\left(N_{c}\right)$ gauge theory with $N_{f}+1$ quarks $Q+\tilde{Q}$ and vanishing superpotential. In the same limit, the fixed point determines the value of the R-charge:

$$
R_{Q}\left(0^{+}\right)=1-\frac{N_{c}}{N_{f}+1} .
$$

In the large $N_{c}$ limit (as we have $N_{f}=\frac{3}{2} N_{c}+1$ ), this value is clearly interacting, $R_{Q} \rightarrow 1 / 3$, in accord with the composite object $Q \cdot \tilde{Q}$ becoming free. We do not know the value of the electric gauge coupling at other values of $t$.

\section{IR $(-\infty<t<0)$ : Magnetic theory}

We now turn to the flow of interest, towards the IR, for $t<0$. Here the magnetic theory is an $\operatorname{SU}\left(\tilde{N}_{c}\right)$ gauge theory with $N_{f}$ quarks $q+\tilde{q}$ and $N_{f} \times N_{f}$ gauge singlet mesons, which for convenience we continue to call $\Phi$. At $t=0$ the boundary conditions of the couplings,

$$
\tilde{\alpha}_{g} \equiv \frac{\tilde{N}_{c} \tilde{g}^{2}}{(4 \pi)^{2}}, \quad \tilde{\alpha}_{y} \equiv \frac{\tilde{N}_{c} \tilde{y}^{2}}{(4 \pi)^{2}},
$$

are determined by continuity, ${ }^{9}$

$$
\begin{aligned}
& \tilde{\alpha}_{g}\left(0^{-}\right)=\frac{\tilde{N}_{c}}{\tilde{N}_{c}+1} \tilde{\alpha}_{g}\left(0^{+}\right), \\
& \tilde{\alpha}_{y}\left(0^{-}\right)=\frac{\tilde{N}_{c}}{\tilde{N}_{c}+1} \tilde{\alpha}_{y}\left(0^{+}\right) .
\end{aligned}
$$

The flow of the magnetic theory can be determined perturbatively from the RGEs. Defining

$$
\begin{aligned}
& \Delta_{\tilde{g}}^{(-)}(t)=\tilde{\alpha}_{g}(t)-\tilde{\alpha}_{g}(-\infty), \\
& \Delta_{\tilde{y}}^{(-)}(t)=\tilde{\alpha}_{y}(t)-\tilde{\alpha}_{y}(-\infty),
\end{aligned}
$$

where the new fixed point is at

$$
\begin{aligned}
& \tilde{\alpha}_{g}(-\infty)=\frac{\left(2 \frac{N_{f}}{\tilde{N}_{c}}+1\right)\left(\frac{N_{f}}{\tilde{N}_{c}}-3\right)}{6+8 \frac{N_{f}}{\tilde{N}_{c}}-4\left(\frac{N_{f}}{\tilde{N}_{c}}\right)^{2}+2 \frac{N_{f}}{\tilde{N}_{c}^{3}}} \stackrel{N_{f} \rightarrow 3 \tilde{N}_{c}-1}{\longrightarrow} \frac{7}{6 \tilde{N}_{c}}+\frac{25}{9 \tilde{N}_{c}^{2}}+\mathcal{O}\left(1 / \tilde{N}_{c}^{3}\right), \\
& \tilde{\alpha}_{y}(-\infty)=\frac{2\left(1-\frac{1}{\tilde{N}_{c}^{2}}\right)\left(\frac{N_{f}}{\tilde{N}_{c}}-3\right)}{6+8 \frac{N_{f}}{\tilde{N}_{c}}-4\left(\frac{N_{f}}{\tilde{N}_{c}}\right)^{2}+2 \frac{N_{f}}{\tilde{N}_{c}^{3}}} \stackrel{N_{f} \rightarrow 3 \tilde{N}_{c}-1}{\longrightarrow} \frac{1}{3 \tilde{N}_{c}}+\frac{8}{9 \tilde{N}_{c}^{2}}+\mathcal{O}\left(1 / \tilde{N}_{c}^{3}\right),
\end{aligned}
$$

they are

$$
\begin{aligned}
& \frac{d \tilde{\alpha}_{g}(t)}{d t}=-2 \tilde{\alpha}_{g}^{2}(t) \times\left[\left(6-4 \frac{N_{f}}{\tilde{N}_{c}}+2 \frac{N_{f}}{\tilde{N}_{c}^{3}}\right) \Delta_{\tilde{g}}^{(-)}(t)+2\left(\frac{N_{f}}{\tilde{N}_{c}}\right)^{2} \Delta_{\tilde{y}}^{(-)}(t)\right], \\
& \frac{d \tilde{\alpha}_{y}(t)}{d t}=2 \tilde{\alpha}_{y}(t) \times\left[-2\left(1-\frac{1}{\tilde{N}_{c}^{2}}\right) \Delta_{\tilde{g}}^{(-)}(t)+\left(2 \frac{N_{f}}{\tilde{N}_{c}}+1\right) \Delta_{\tilde{y}}^{(-)}(t)\right] .
\end{aligned}
$$

The evolution is shown in Fig. 2 for $\tilde{N}_{c}=100$ and $N_{f}=3 \tilde{N}_{c}-1$.

It is useful to explicitly express the flowing $R$-charges in terms of the couplings. This we can do because the theory is perturbative (approximately, order by order in perturbation theory). From the usual definition of the NSVZ beta function and the relation in $(2.22)$, we have

$$
\begin{aligned}
& \beta\left(\tilde{\alpha}_{g}\right)=-6 \tilde{\alpha}_{g}^{2} f\left(\tilde{\alpha}_{g}\right)\left(1+\frac{N_{f}}{\tilde{N}_{c}}\left(R_{q}-1\right)\right), \\
& \beta\left(\tilde{\alpha}_{y}\right)=3 \tilde{\alpha}_{y}\left(2 R_{q}+R_{\Phi}-2\right),
\end{aligned}
$$

\footnotetext{
${ }^{9}$ Note that we are using the NSVZ scheme which is a mass-independent scheme: this means that the perturbative gauge couplings are continuous passing the mass scale, while the beta functions or anomalous dimensions are not. The apparent discontinuity in (3.13) is clear from the way the coupling constants are defined in (3.12), i.e., with the discontinuity being in the number of colors.
} 


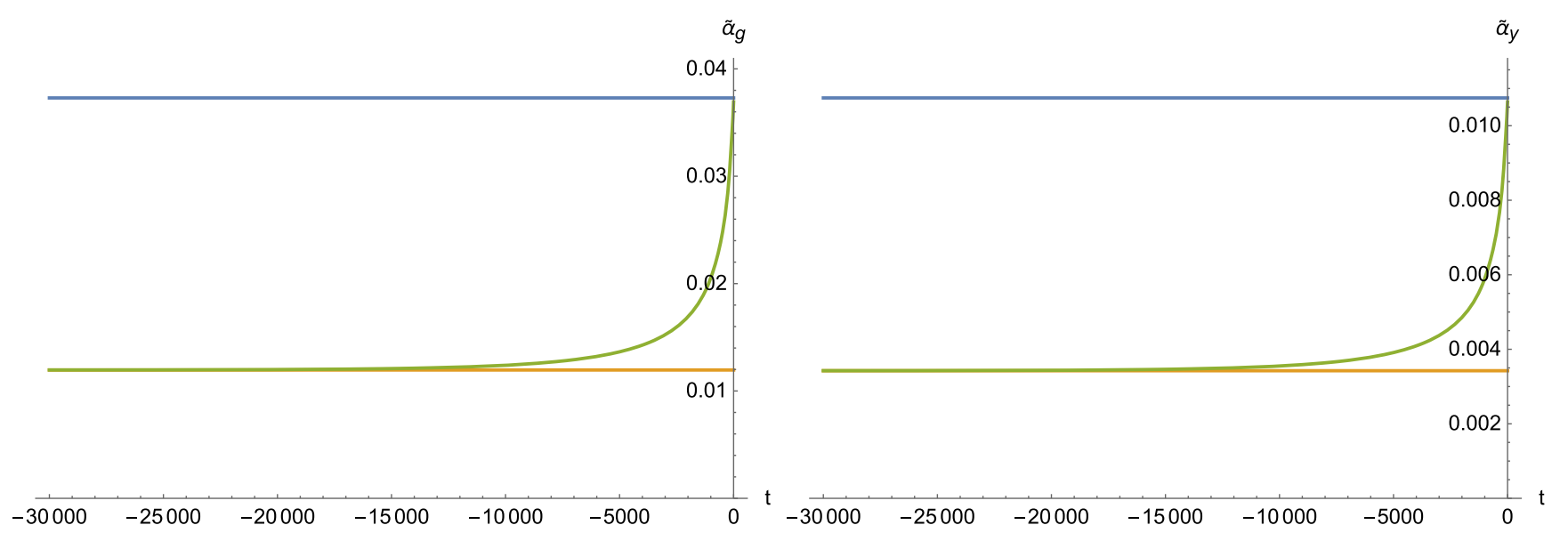

FIG. 2. The perturbative running of the gauge (left) and Yukawa (right) coupling constants of the magnetic theory for $\tilde{N}_{c}=100$ and $N_{f}=3 \tilde{N}_{c}-1$, from the UV fixed point ( $t=0$ with $N_{f}+1$ quarks and $\tilde{N}_{c}+1$ colors), to the IR fixed point $\left(t=-\infty\right.$ with $N_{f}$ quarks and $\tilde{N}_{c}$ colors). The lower and upper lines denote the UV and IR values of the couplings.

with

$$
f(x) \equiv \frac{1}{1-2 x}
$$

Comparison with the rhs of (3.16) gives

$$
\begin{aligned}
& R_{q}(t)-R_{q}(-\infty)=-\frac{2}{3}\left(1-\frac{1}{\tilde{N}_{c}^{2}}\right) \Delta_{\tilde{g}}^{(-)}+\frac{2 N_{f}}{3} \frac{\tilde{N}_{c}}{(-)}+\mathcal{O}\left(\Delta^{2}\right) \\
& R_{\Phi}(t)-R_{\Phi}(-\infty)=\frac{2}{3} \Delta_{\tilde{y}}^{(-)}+\mathcal{O}\left(\Delta^{2}\right)
\end{aligned}
$$

Their evolution for $t<0$ is shown in Fig. 3. Note that although we do not display them explicitly, the order $\Delta^{2}$ terms as derived from Eq. (3.17) are actually required later in order to get consistent convergence to the IR fixed point in the strongly coupled description.

\section{IR $(-\infty<t<0)$ : Electric theory}

Up to this point, for $t<0$, everything has been perturbative. Now let us now consider the original electric theory in the range $-\infty<t<0$. In the limit $t \rightarrow-\infty$, the theory is $\mathrm{SU}\left(N_{c}\right) \mathrm{SQCD}$ with $N_{f}$ quarks $Q+\tilde{Q}$ and no superpotential.

Let us assume that the same pair of dual theories describe the physics along the whole RGE running. As the parameter $a_{K S}$ is a function of the amplitude its definition is independent of which description is being used and, hence, its value in the electric and magnetic theories is the same all along the flow.

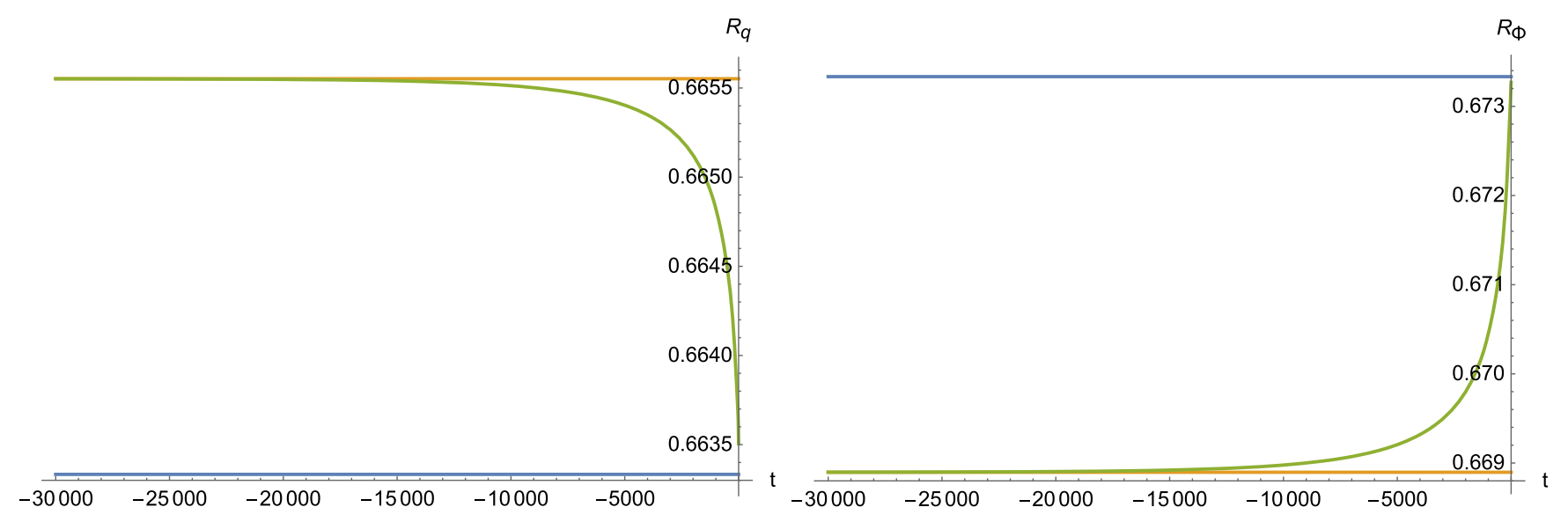

FIG. 3. The "flowing" R-charges (green) of the quark (left and meson (right) in the magnetic SQCD with gauge SU $\left(\tilde{N}_{c}\right)$ and $N_{f}$ quarks $q+\tilde{q}$ and $N_{f}^{2}$ mesons $\Phi$, with $N_{f}=3 \tilde{N}_{c}-1$. The flow has been found using the perturabative relations (3.17) and (3.17) and using $\tilde{N}_{c}=100$. The blue straight lines are the values $R_{q}\left(0^{+}\right)$and $R_{\Phi}\left(0^{+}\right)$obtained in the fixed point above the mass $m$. Notice that the values $R_{q}\left(0^{-}\right)$and $R_{\Phi}\left(0^{-}\right)$do not coincide with them: although the gauge couplings $\tilde{g}$ and $\tilde{y}$ are continuous, the $R$-charges are not: they are in some sense proportional to the noncontinuous beta functions. Finally the orange straight lines are the limiting values $R_{q}(-\infty)$ and $R_{\Phi}(-\infty)$ obtained from the IR fixed point couplings. 


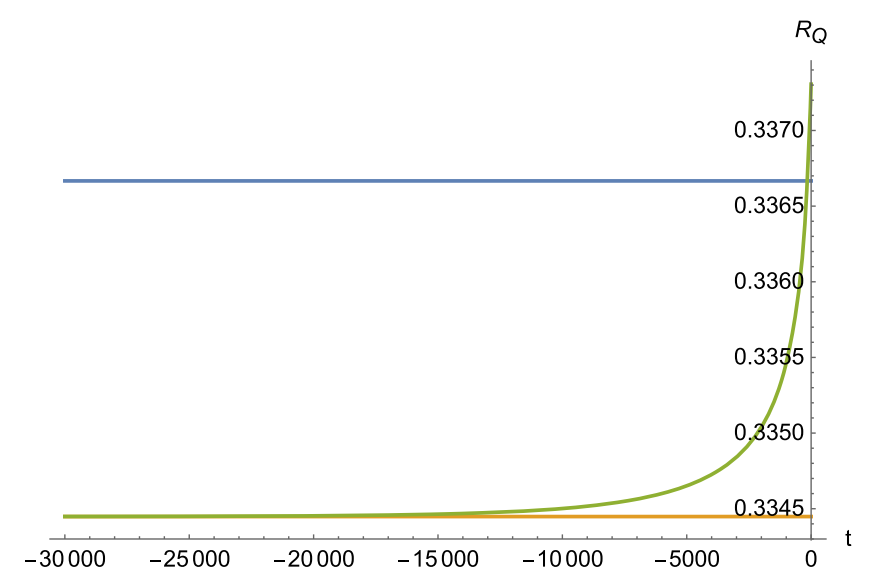

FIG. 4. The R-charge (green) of the quark in the electric SQCD with gauge group $\mathrm{SU}\left(N_{c}\right)$ and $N_{f}$ quarks $Q+\tilde{Q}$, with $N_{f}=3 \tilde{N}_{c}-1$, using (3.20). As before, the blue straight line is the value at $t=0^{+}$, while the orange line is the asymptotic value in the IR.

We will adopt the assumption, motivated in the Introduction, that in regions where the beta functions are small the $a_{K S}$-function is the same as the function $a_{K}$ derived using the Lagrange multiplier definition [17]. Hence, using (2.21) and equating $a_{K}$ 's in the two descriptions as in the Appendix, one finds

$$
\begin{aligned}
2\left(N_{c}^{2}-1\right)+2 N_{f} N_{c}\left(a_{1}\left(R_{Q}(t)\right)\right. & \\
& \left.-\left(R_{Q}(t)-R_{Q}(-\infty)\right) a_{1}^{\prime}\left(R_{Q}(t)\right)\right) \\
= & 2\left(\tilde{N}_{c}^{2}-1\right)+2 N_{f} \tilde{N}_{c}\left(a_{1}\left(R_{q}(t)\right)\right. \\
& -\left(R_{q}(t)-R_{q}(-\infty)\right) a_{1}^{\prime}\left(R_{q}(t)\right)+N_{f}^{2}\left(a_{1}\left(R_{\Phi}(t)\right)\right. \\
& \left.-\left(R_{\Phi}(t)-R_{\Phi}(-\infty)\right) a_{1}^{\prime}\left(R_{\Phi}(t)\right)\right),
\end{aligned}
$$

which can be used to determine $R_{Q}(t)$. Its behavior is shown in Fig. 4.

From there it is straightforward to determine the gauge coupling from the NSVZ beta function. Defining the electric gauge coupling as

$$
\alpha_{g} \equiv \frac{N_{c} g^{2}}{(4 \pi)^{2}}
$$

and using

$$
\beta\left(\alpha_{g}\right)=-6 \alpha_{g}^{2} f\left(\alpha_{g}\right)\left(1+\frac{N_{f}}{N_{c}}\left(R_{Q}-1\right)\right),
$$

one can now integrate, to find

$$
F\left(\alpha_{g}(t)\right)-F\left(\alpha_{g}\left(0^{-}\right)\right)=\int_{0}^{t} d t^{\prime}\left(1+\frac{N_{f}}{N_{c}}\left(R_{Q}\left(t^{\prime}\right)-1\right)\right),
$$

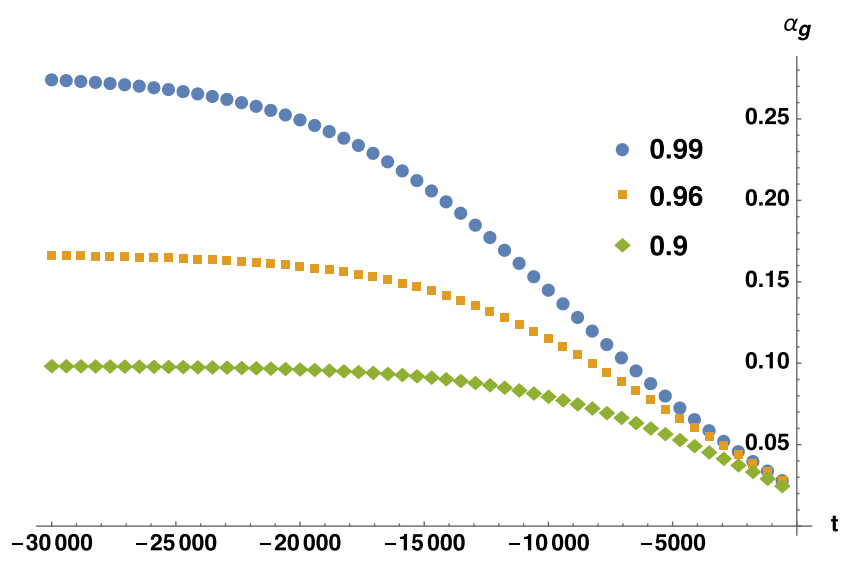

FIG. 5. The nonperturbative running of the gauge coupling constant of the electric theory $\mathrm{SU}\left(N_{c}\right)$ with $N_{f}$ quarks $Q+\tilde{Q}$, for three different values of the ratio $\alpha_{g}\left(0^{-}\right) / \alpha_{g}^{\max }\left(0^{-}\right)$, and for $\tilde{N}_{c}=100, N_{f}=3 \tilde{N}_{c}-1$ and $N_{c}=N_{f}-\tilde{N}_{c}$. Note that $\alpha_{g} \equiv \frac{N_{c} g^{2}}{(4 \pi)^{2}}$.

where

$$
F(x) \equiv \frac{1}{6}\left(\frac{1}{x}+2 \log x\right)
$$

This can then be solved for $\alpha_{g}$. Note that as mentioned the $\mathcal{O}\left(\Delta^{2}\right)$ terms in Eq. (3.19) are required here. If they are omitted then there are order $1 / N_{c}^{2}$ errors in the integrand, which over the order $-t \sim N_{c}^{2}$ running required to get to the fixed point, translates into errors of order unity: in other words there would not be proper convergence to a fixed point.

Of course, we do not know the numerical value of the boundary condition, $\alpha_{g}\left(0^{-}\right)$, in the electric theory, but since the rhs of (3.23) is negative, and since the gauge coupling must obey $\alpha_{g}<1 / 2$ in order that $f\left(\alpha_{g}\right)$ defined in (3.18) does not change sign, there is a maximum allowed value of $\alpha_{g}\left(0^{-}\right)$given by

$$
F\left(\alpha_{g}^{\max }\left(0^{-}\right)\right)+\int_{0}^{-\infty} d t^{\prime}\left(1+\frac{N_{f}}{N_{c}}\left(R_{Q}\left(t^{\prime}\right)-1\right)\right)=F(1 / 2) .
$$

For our inputs, this is given by

$$
\alpha_{g}^{\max }\left(0^{-}\right)=0.0216164 .
$$

As an illustrative example, we take three different inputs $(0.99,0.96,0.9)$ for the ratio $\alpha_{g}\left(0^{-}\right) / \alpha_{g}^{\max }\left(0^{-}\right)$and obtain numerically the flows shown in Fig. 5 for the nonperturbative coupling $\alpha_{g}(t)$. There is, of course, only one correct numerical boundary condition at $t=0^{-}$corresponding to the electric theory dual to the perturbative magnetic one, 
but unfortunately it cannot be determined. ${ }^{10}$ All we know is that it must be nonperturbative, so too small ratios $\alpha_{g}\left(0^{-}\right) / \alpha_{g}^{\max }\left(0^{-}\right)$are unacceptable because they would not reproduce the known anomalous dimensions in the deep IR.

The entire flow including the $R$-charges can, of course, be expressed in terms of the Lagrange multipliers of [17], in the manner described in the Introduction and in Sec. II. We included them for completeness in the Appendix.

\section{EQUALITY OF CRITICAL EXPONENTS}

The critical exponent provides a mild but nevertheless important check on the consistency of this picture. It is defined as the minimal eigenvalue of the matrix of coupling derivatives of the beta functions around the fixed point $^{11}$ :

$$
\beta^{\prime} \equiv \min \left\{\text { positive eigenvalues }\left(\frac{\partial \beta_{\alpha_{a}}}{\partial \alpha_{b}}\right)_{\text {F.P. }}\right\}
$$

It is a renormalization-scheme-independent quantity and, therefore, should be equal for dual theories $[25,26]$. Usually, of course, this equivalence cannot be checked because one cannot compute in the strongly coupled theory. However, our prescription $\left(a_{K S}^{(e l)}=a_{K S}^{(\mathrm{mag})}\right)$ allows it to be checked explicitly, as we now show.

In the magnetic description, the theory is perturbative, and so we can simply use (3.16) to evaluate the critical exponent:

$$
\left(\frac{\partial \beta_{\alpha_{a}}}{\partial \alpha_{b}}\right)_{\text {F.P. }}=\left(\begin{array}{cc}
-2 \tilde{\alpha}_{g}^{2}(-\infty)\left(6-4 \frac{N_{f}}{\tilde{N}_{c}}+2 \frac{N_{f}}{\tilde{N}_{c}^{3}}\right) & -2 \tilde{\alpha}_{g}^{2}(-\infty)\left(2\left(\frac{N_{f}}{\tilde{N}_{c}}\right)^{2}\right) \\
2 \tilde{\alpha}_{y}(-\infty)\left(1-\frac{1}{\tilde{N}_{c}^{2}}\right)(-2) & 2 \tilde{\alpha}_{y}(-\infty)\left(2 \frac{N_{f}}{\tilde{N}_{c}}+1\right)
\end{array}\right)
$$

For $N_{f}=3 \tilde{N}_{c}-1$, one obtains the leading order in $1 / \tilde{N}_{c}$ approximation, using (3.15),

$$
\beta_{\mathrm{mag}}^{\prime}=\frac{7}{3 \tilde{N}_{c}^{2}}
$$

while the second, larger, eigenvalue is found to be equal to $14 /\left(3 \tilde{N}_{c}\right)$.

In the strongly coupled electric description, there is a single gauge coupling, so that

$$
\beta_{e l}^{\prime}=\left.\frac{\partial \beta_{\alpha_{g}}(t)}{\partial \alpha_{g}(t)}\right|_{t \rightarrow-\infty}=\left.\frac{\frac{d}{d t} \beta_{\alpha_{g}}(t)}{\frac{d}{d t} \alpha_{g}(t)}\right|_{t \rightarrow-\infty}=\left.\frac{d}{d t} \log \left(R_{Q}(t)-R_{Q}(-\infty)\right)\right|_{t \rightarrow-\infty} .
$$

Usually, in the nonperturbative theory, the relation between $R_{Q}(t)$ and $\alpha_{g}(t)$ is not known. Here, however, we have a relation between $R_{Q}(t)$ and the known $R_{q}(t)$ and $R_{\Phi}(t)$ of the perturbative magnetic theory through (3.20). We may, therefore, expand $a$ around $t=-\infty$,

$$
a(t)=a(-\infty)+\sum_{i, j} \frac{1}{2} \frac{\partial^{2} a}{\partial R_{i} \partial R_{j}}(-\infty)\left(R_{i}(t)-R_{i}(-\infty)\right)\left(R_{j}(t)-R_{j}(-\infty)\right)+\cdots,
$$

and from there must find

$$
\frac{\partial^{2} a_{e l}}{\partial R_{Q}^{2}}(-\infty)\left(R_{Q}(t)-R_{Q}(-\infty)\right)^{2} \approx \frac{\partial^{2} a_{\mathrm{mag}}}{\partial R_{q}^{2}}(-\infty)\left(R_{q}(t)-R_{q}(-\infty)\right)^{2}+\frac{\partial^{2} a_{\mathrm{mag}}}{\partial R_{M}^{2}}(-\infty)\left(R_{\Phi}(t)-R_{\Phi}(-\infty)\right)^{2} .
$$

Since the second derivative of $a$ over the $R$-charges is proportional to the $b$-central charge of a conserved current (in this case it is the baryon current) and, thus, strictly nonzero, we must have the same scaling,

$$
R_{Q}(t)-R_{Q}(-\infty) \sim R_{q}(t)-R_{q}(-\infty) \sim R_{\Phi}(t)-R_{\Phi}(-\infty) \sim \exp \left(\beta_{\text {mag }}^{\prime} t\right),
$$

in the asymptotic region $t \rightarrow-\infty$. But then, from (4.4), we consistently find

$$
\beta_{e l}^{\prime}=\beta_{\mathrm{mag}}^{\prime} .
$$

\footnotetext{
${ }^{10}$ It would be interesting to attempt to extend the approach to include the mass term explicitly with another Lagrange multiplier, as for the free-field theory in the Introduction. However, as one would have to describe a Higgsing in the magnetic theory, this would be significantly more complicated, and it is not clear how one could fix several Lagrange multipliers with only a single $a$ parameter.

${ }^{11}$ Here one cannot compare the full matrices or even all their eigenvalues, because e.g., the dimensions of the matrices do not agree. However the minimal eigenvalue has a physical scheme-independent meaning in both descriptions.
} 
We conclude that $a_{e l}=a_{\mathrm{mag}}$ along the flow is compatible with the equality of the electric and magnetic critical exponents. Of course, this is not a particularly restrictive condition, and many other relations would have given equality. For example

$$
a_{\mathrm{mag}}=A\left(a_{e l}\right)
$$

for an arbitrary function $A(x)$ with

$$
\begin{aligned}
A\left(a_{e l}(-\infty)\right) & =a_{e l}(-\infty), \\
A^{\prime}\left(a_{e l}(-\infty)\right) & \neq 0 .
\end{aligned}
$$

would suffice.

\section{CONCLUSION}

In this paper, we discussed the use of the $a$ central charge as a method of determining the flow in a strongly coupled supersymmetric theory from its weakly coupled dual. Although there are other examples of exact duality in field theory along an entire flow (e.g., [27]), this method seems particularly general and well suited to $\mathcal{N}=1$ supersymmetry. Crucial to the approach is the equivalence of the scale-dependent $a$ parameter determined from the fourdilaton amplitude with an IR cutoff, and the $a$ parameter determined in the Lagrange multiplier method of Refs. $[17,18]$ with "flowing" $R$-charges. We showed that this equivalence holds directly for massive free $\mathcal{N}=1$ superfields, as well as weakly coupled SQCD. Assuming it to hold generally amounts to a particularly physical choice of RG scheme, in which the running $R$-charges are always determined precisely from the four-dilaton amplitude. In this scheme, which is clearly well defined regardless of which formulation is being used, one can map the flow of a weakly coupled magnetic dual to the original strongly coupled electric theory. The specific system we considered was the well-known pair of original SQCD Seiberg duals, with the magnetic description (with weak gauge and Yukawa coupling constants) running perturbatively from a fixed point in the UV to a different fixed point in the IR due to a mass-deformation, and the electric SQCD dual running between strongly coupled fixed points due to a meson-induced Higgsing.

We should add that the mapping only seems to work straightforwardly in the direction of magnetic to electric, as in that case there is only one $R$-charge to determine (namely that of the electric quarks), and there is only one parameter (namely, the $a$ parameter) with which to do it. Mapping in the converse direction may be possible in conjunction with $a$ maximization [28], but is less obvious.

\section{ACKNOWLEDGMENTS}

We are extremely grateful to Colin Poole for interesting discussions. B. B. acknowledges the financial support from the Slovenian Research Agency (research core funding No. P1-0035). The work of F. S. is partially supported by the Danish National Research Foundation under the Grant No. DNRF:90. B. B. thanks CP3 Origins Odense for hospitality.

\section{APPENDIX: THE LAGRANGE MULTIPLIERS}

Here, we explicitly show how the Lagrange multipliers of $[17,18]$ flow in the model discussed in Sec. III. We start with the original magnetic $a$ function,

$$
\begin{aligned}
\tilde{a}_{\mathrm{mag}}= & 2\left(\tilde{N}_{c}^{2}-1\right)+2 \tilde{N}_{c} N_{f} a_{1}\left(R_{q}\right)+N_{f}^{2} a_{1}\left(R_{\Phi}\right) \\
& -\tilde{\lambda}_{g} N_{f}\left(R_{q}-R_{q}(-\infty)\right)+\tilde{\lambda}_{y} N_{f}\left(2\left(R_{q}-R_{q}(-\infty)\right)\right. \\
& \left.+\left(R_{\Phi}-R_{\Phi}(-\infty)\right)\right) .
\end{aligned}
$$

By a maximization, we have [28]

$$
\frac{\partial \tilde{a}_{\mathrm{mag}}}{\partial R_{q}}=\frac{\partial \tilde{a}_{\mathrm{mag}}}{\partial R_{\Phi}}=0,
$$

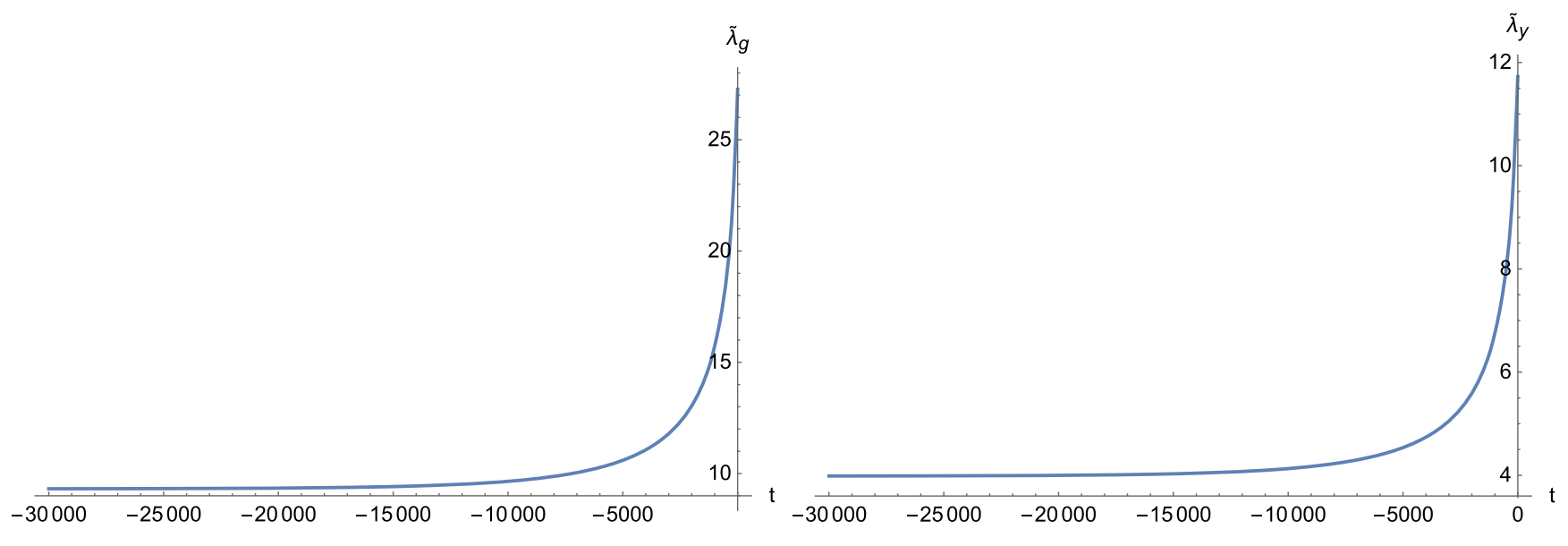

FIG. 6. The Lagrange multipliers of the magnetic theory. 
which gives the following for the Lagrange multipliers:

$$
\begin{gathered}
\tilde{\lambda}_{g}=2 \tilde{N}_{c} a_{1}^{\prime}\left(R_{q}\right)-2 N_{f} a_{1}^{\prime}\left(R_{\Phi}\right), \\
\tilde{\lambda}_{y}=-N_{f} a_{1}^{\prime}\left(R_{\Phi}\right) .
\end{gathered}
$$

Similarly, for the electric theory, one gets

$$
\begin{aligned}
\tilde{a}_{e l}= & 2\left(N_{c}^{2}-1\right)+2 N_{c} N_{f} a_{1}\left(R_{Q}\right) \\
& -\lambda_{g} N_{f}\left(R_{Q}-R_{Q}(-\infty)\right),
\end{aligned}
$$

giving

$$
\lambda_{g}=2 N_{c} a_{1}^{\prime}\left(R_{Q}\right)
$$

Plugging (A3) and (A4) into (A1), (A6) into (A5), and equating the two $a$-central charges, we obtain (3.20).

From the perturbative knowledge of $R_{q}(t)$ and $R_{\Phi}(t)$, we can thus draw $\tilde{\lambda}_{g}(t)$ in the magnetic theory discussed in the

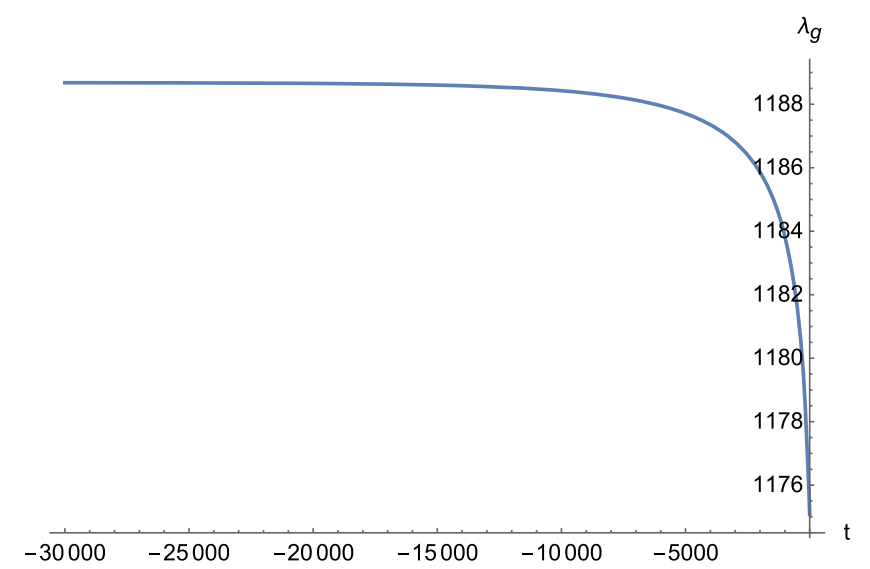

FIG. 7. The Lagrange multiplier of the electric theory.

main text, while from the nonperturbative knowledge of $R_{Q}(t)$ using (3.20) we get $\lambda_{g}(t)$ for the strongly coupled electric theory. The graphs are shown in Figs. 6 and 7.
[1] A. B. Zamolodchikov, 'Irreversibility' of the flux of the renormalization group in a 2-D field theory, Pis'ma Zh. Eksp. Teor. Fiz. 43, 565 (1986) [JETP Lett. 43, 730 (1986)].

[2] J. L. Cardy, Is there A C theorem in four-dimensions, Phys. Lett. B 215, 749 (1988).

[3] Z. Komargodski and A. Schwimmer, On renormalization group flows in four dimensions, J. High Energy Phys. 12 (2011) 099.

[4] M. A. Luty, J. Polchinski, and R. Rattazzi, The $a$-theorem and the asymptotics of 4D quantum field theory, J. High Energy Phys. 01 (2013) 152.

[5] F. Baume, B. Keren-Zur, R. Rattazzi, and L. Vitale, The local Callan-Symanzik equation: Structure and applications, J. High Energy Phys. 08 (2014) 152.

[6] R. Auzzi and B. Keren-Zur, Superspace formulation of the local RG equation, J. High Energy Phys. 05 (2015) 150.

[7] H. Osborn, Derivation of a four-dimensional $c$ Theorem, Phys. Lett. B 222, 97 (1989).

[8] I. Jack and H. Osborn, Analogs for the $c$ theorem for fourdimensional renormalizable field theories, Nucl. Phys. B343, 647 (1990).

[9] H. Osborn, Weyl consistency conditions and a local renormalization group equation for general renormalizable field theories, Nucl. Phys. B363, 486 (1991).

[10] G. M. Shore, The c and a-theorems and the local renormalisation group, arXiv:1601.06662.

[11] O. Antipin, M. Mojaza, C. Pica, and F. Sannino, Magnetic fixed points and emergent supersymmetry, J. High Energy Phys. 06 (2013) 037.

[12] N. Seiberg, Electric - magnetic duality in supersymmetric non-Abelian gauge theories, Nucl. Phys. B435, 129 (1995).
[13] K. A. Intriligator and N. Seiberg, Lectures on supersymmetric gauge theories and electric-magnetic duality, Nucl. Phys. B Proc. Suppl. 45, 1 (1996); Subnuclear series 34, 237 (1997).

[14] Z. Komargodski, The constraints of conformal symmetry on RG flows, J. High Energy Phys. 07 (2012) 069.

[15] See C. Scrucca, Advanced quantum field theory, Doctoral School in Physics, EPFL, https://iphys.epfl.ch/page141204-en.html.

[16] S. Abel and A. Mariotti, Novel Higgs potentials from gauge mediation of exact scale breaking, Phys. Rev. D 89, 125018 (2014).

[17] D. Kutasov, New results on the 'A theorem' in fourdimensional supersymmetric field theory, arXiv:hep-th/ 0312098.

[18] D. Kutasov and A. Schwimmer, Lagrange multipliers and couplings in supersymmetric field theory, Nucl. Phys. B702, 369 (2004).

[19] D. Kutasov, A. Parnachev, and D. A. Sahakyan, Central charges and $\mathrm{U}(1)(\mathrm{R})$ symmetries in $\mathcal{N}=1$ super YangMills, J. High Energy Phys. 11 (2003) 013.

[20] B. A. Kniehl, Dispersion relations in loop calculations, Acta Phys. Pol. B 27, 3631 (1996).

[21] D. Anselmi, D.Z. Freedman, M. T. Grisaru, and A. A. Johansen, Nonperturbative formulas for central functions of supersymmetric gauge theories, Nucl. Phys. B526, 543 (1998).

[22] D. Anselmi, J. Erlich, D. Z. Freedman, and A. A. Johansen, Positivity constraints on anomalies in supersymmetric gauge theories, Phys. Rev. D 57, 7570 (1998).

[23] M. J. Strassler, The duality cascade, arXiv:hep-th/0505153. 
[24] S. P. Martin and M. T. Vaughn, Two loop renormalization group equations for soft supersymmetry breaking couplings, Phys. Rev. D 50, 2282 (1994); Erratum, Phys. Rev. D 78, 039903 (2008).

[25] D. Anselmi, M. T. Grisaru, and A. Johansen, A critical behavior of anomalous currents, electric-magnetic universality and CFT in four-dimensions, Nucl. Phys. B491, 221 (1997).

[26] E. Barnes, K. A. Intriligator, B. Wecht, and J. Wright, Evidence for the strongest version of the 4D A-theorem, via $a$-maximization along RG flows, Nucl. Phys. B702, 131 (2004).

[27] A. Kapustin and M. J. Strassler, On mirror symmetry in three-dimensional abelian gauge theories, J. High Energy Phys. 04 (1999) 021.

[28] K. A. Intriligator and B. Wecht, The exact superconformal $\mathrm{R}$ symmetry maximizes $a$, Nucl. Phys. B667, 183 (2003). 